\title{
BIORTHOGONALITY AND INTEGRATION
}

\author{
SAMUEL KAPLAN
}

1. Introduction. Practically all the work done to date on biorthogonality, as can be seen from Dieudonné's paper [1], has been confined to countable systems. An obvious generalization is to put no restriction on the cardinality of the two biorthogonal sets while leaving the definition unchanged otherwise $[1$, p. 7$]$. However, there is a kind of biorthogonality occurring in integration which does not satisfy this definition. In the present note, we give a generalization which includes both integration of bounded functions and the above generalization.

Most of the material, and especially the proof of the deep part of the theorem, is taken from Dieudonné's paper, with the minor modifications needed for the present case.

2. We consider a real Banach space $E$. If $x \in E$ and $y \in E^{\prime}$ (the dual space), the value $y(x)$ will be denoted alternatively by $x \cdot y$.

We define a partial order $\prec$ on the finite subsets of $E$ as follows: If $X_{1}, X_{2}$ are two finite subsets, then $X_{1} \prec X_{2}$ will mean that $X_{1}$ is contained in the linear subspace generated by $X_{2}$.

We shall call two subsets $X=\{\xi\}$ of $E$ and $\Upsilon=\{\eta\}$ of $E^{\prime}$ biorthogonal if they satisfy the following conditions (I) and (II):

(I) (a) For each pair $\xi, \eta, \xi \cdot \eta=0$ or 1 ;

(b) No $\xi$ has value 0 on every $\eta$; and no $\eta$ has value 0 on every $\xi$.

A finite subset $X=\left\{\xi_{1}, \cdots, \xi_{k}\right\}$ of $X$ will be called a $\Upsilon$-orthogonal set if no $\eta$ has value 1 on more than one $\xi_{i}, i=1, \cdots, k$.

(II) The $\Upsilon$-orthogonal sets of $X$ form a directed system under $<$.

In the remainder of the paper we shall assume a fixed biorthogonal pair of sets $\mathcal{X}, \Upsilon$, with elements denoted by $\xi, \eta$ respectively. We remark that each individual $\xi$ is a $\Upsilon$-orthogonal set.

Proposition 1. Given any $\Upsilon$-orthogonal set $X=\left\{\xi_{1}, \cdots, \xi_{k}\right\}$ of $X$, there exists a subset $Y=\left\{\eta_{1}, \cdots, \eta_{k}\right\}$ of $\Upsilon$ which is biorthogonal to $X$ (in the ordinary sense).

From (I), for each $i=1, \cdots, k$, there is an $\eta_{i}$ such that $\xi_{i} \cdot \eta_{i}=1$.

Received by the editors January 17, 1955 and, in revised form, March 31, 1955. 
Then, by the definition of $\Upsilon$-orthogonal set, $\left\{\eta_{1}, \cdots, \eta_{k}\right\}$ is biorthogonal to $X$.

COROLLARY. The elements of each $\Upsilon$-orthogonal set are linearly independent.

Proposition 2. Given any finite subset $Y=\left\{\eta_{1}, \cdots, \eta_{k}\right\}$ of $\Upsilon$, there exists a $\Upsilon$-orthogonal set $X=\left\{\xi_{1}, \cdots, \xi_{k}\right\}$ which is biorthogonal to $Y$.

From (I), for each $i=1, \cdots, k$, there is a $\xi_{i}^{\prime}$ such that $\xi_{i}^{\prime} \cdot \eta_{i}=1$. Since each $\xi_{i}^{\prime}$ is a $\Upsilon$-orthogonal set, it follows from (II) that there is a $\Upsilon$-orthogonal set $\left\{\xi_{1}, \cdots, \xi_{m}\right\}$ which is $>\xi_{i}^{\prime}$ for every $i=1, \cdots, k$. Each $\eta_{i}$ has value 1 on exactly one $\xi_{j}$, which we denote by $\xi_{j_{i}}$. The set $\left\{\xi_{j_{1}}, \cdots, \xi_{j_{k}}\right\}$ is then the required $X$.

COROLlary. The elements of $\Upsilon$ are linearly independent.

Henceforth we shall reserve the letter $X$ to denote a $\Upsilon$-orthogonal set of $\mathcal{X}$, and $Y$ to denote a finite subset of $\Upsilon$.

Suppose $x$ is a linear combination of $\xi$ 's. It follows from (II) that $x$ is a linear combination of the elements of some $X_{0}$, and hence of every $X>X_{0}$. Consider any such $X=\left\{\xi_{1}, \cdots, \xi_{k}\right\}>X_{0}$. If we arbitrarily choose a set $Y=\left\{\eta_{1}, \cdots, \eta_{k}\right\}$ biorthogonal to $X$, we have, of course, $x=\sum_{1}^{k}\left(x \cdot \eta_{i}\right) \xi_{i}$. For later use we state this formally:

Proposition 3. Let $x$ be a linear combination of $\xi$ 's. There exists an $X_{0}$ such that if $X=\left\{\xi_{1}, \cdots, \xi_{k}\right\}$ is $>X_{0}$ and $Y=\left\{\eta_{1}, \cdots, \eta_{k}\right\}$ is biorthogonal to $X$, then $x=\sum_{1}^{k}\left(x \cdot \eta_{i}\right) \xi_{i}$.

From now on, whenever we write the expressions $\sum_{1}^{k}\left(x \cdot \eta_{i}\right) \xi_{i}$ or $\sum_{1}^{k}\left(\xi_{i} \cdot y\right) \eta_{i}$, it will be understood that the sets $\left\{\xi_{1}, \cdots, \xi_{k}\right\}$ and $\left\{\eta_{1}, \cdots, \eta_{k}\right\}$ are biorthogonal.

Proposition 4. The following three conditions on the system $(X, \Upsilon)$ are equivalent:

(a) For each $x \in E$, there is a positive number $M$ (dependent on $x$ ) such that $\left\|\sum_{1}^{k}\left(x \cdot \eta_{i}\right) \xi_{i}\right\| \leqq M$ for all biorthogonal pairs $X, Y$;

(b) For each $y \in E^{\prime}$, there is a positive number $N$ (dependent on $y$ ) such that $\left\|\sum_{1}^{k}\left(\xi_{i} \cdot y\right) \eta_{i}\right\| \leqq N$ for all biorthogonal pairs $X, Y$;

(c) For each $x \in E$ and $y \in E^{\prime}$, there is a positive number $P$ (dependent on $x$ and $y$ ) such that $\left|\sum_{1}^{k}\left(\xi_{i} \cdot y\right)\left(x \cdot \eta_{i}\right)\right| \leqq P$ for all biorthogonal pairs $X, Y$.

In $E$, boundedness in the norm and boundedness in the topology $\sigma\left(E, E^{\prime}\right)$ (the weak topology defined on $E$ by $E^{\prime}$ ) are equivalent 
[4, p. 80, Théorème 6]; hence (a) is equivalent to (c). In $E^{\prime}$, boundedness in the norm and boundeness in $\sigma\left(E^{\prime}, E\right)$ are equivalent $[4$, p. 80 , Théorème 5 ]; hence $(b)$ is equivalent to $(c)$.

If the system $(X, Y)$ satisfies any one (hence all three) of the above conditions, we shall call it quasi-regular.

CoROLLARY. If the system $(X, \Upsilon)$ is quasi-regular, then the conditions are satisfied for every $x \in E^{\prime \prime}$.

In $E^{\prime}$, boundedness in the norm and boundedness in $\sigma\left(E^{\prime}, E^{\prime \prime}\right)$ are equivalent; hence it follows from (b) that (c) holds for $x \in E^{\prime \prime}$. In $E^{\prime \prime}$, boundedness in the norm is equivalent to boundedness in $\sigma\left(E^{\prime \prime}, E^{\prime}\right)$; hence (a) also holds for $x \in E^{\prime \prime}$. This establishes the corollary.

Consider an element $x$ of $E$ and a $\Upsilon$-orthogonal set $X=\left\{\xi_{1}, \cdots\right.$, $\left.\xi_{k}\right\}$. Each set $Y=\left\{\eta_{1}, \cdots, \eta_{k}\right\}$ biorthogonal to $X$ assigns to the pair $x, X$ an element of $E, \sum_{1}^{k}\left(x \cdot \eta_{i}\right) \xi_{i}$. Since there are in general many $Y$ 's biorthogonal to $X$, there are in general many such elements of $E$ associated with $x$ and $X$. Let us denote the set of all these elements of $E$ by $S(x, X)$. Letting $x$ vary over $E$ and $X$ over the $\Upsilon$ orthogonal sets, we have that $S(x, X)$ is a many-valued function of $x$ and $X$, with values in $E$. Now for fixed $x$, it is a function of $X$, and since the $X$ 's form a directed set under $\prec$ we can define the MooreSmith limit: The statement $x_{1}=\lim \prec S(x, X)$ ( $x$ fixed)-in either the norm or weak topology - will mean that given any neighborhood $U$ of $x_{1}$, there exists an $X_{0}$ such that $X>X_{0}$ implies $S(x, X) \subset U$. In practice, we shall use the notation $\lim \prec \sum_{1}^{k}\left(x \cdot \eta_{i}\right) \xi_{i}=x_{1}$, or, in case of the norm, $\lim \prec\left\|x_{1}-\sum_{1}^{k}\left(x \cdot \eta_{i}\right) \xi_{i}\right\|=0$.

Similarly, given an element $y$ of $E^{\prime}$ and a $\Upsilon$-orthogonal set $X=\left\{\xi_{1}, \cdots, \xi_{k}\right\}$, each set $Y=\left\{\eta_{1}, \cdots, \eta_{k}\right\}$ biorthogonal to $X$ assigns to the pair $y, X$ an element of $E^{\prime}, \sum_{1}^{k}\left(\xi_{i} \cdot y\right) \eta_{i}$. As above we obtain a many-valued function of $y$ and $X, y$ varying over $E^{\prime}, X$ over the $\Upsilon$-orthogonal sets, with values in $E^{\prime}$. And as above we can talk about $\lim \prec \sum_{1}^{k}\left(\xi_{i} \cdot y\right) \eta_{i}$ (y fixed).

A subset of a topological linear space will be called total if the closed linear subspace generated by it is the entire space.

THEOREM. Let the system $(X, \Upsilon)$ be quasi-regular. Then, of the following properties, (1), (2), (3), (4) are equivalent, and imply (5):

(1) $X$ is total;

(2) For each $x \in E, \lim <\left\|x-\sum_{1}^{k}\left(x \cdot \eta_{i}\right) \xi_{i}\right\|=0$;

(3) For each $x \in E, \lim \prec \sum_{1}^{k}\left(x \cdot \eta_{i}\right) \xi_{i}=x$ in $\sigma\left(E, E^{\prime}\right)$;

(4) For each $y \in E^{\prime}, \lim <\sum_{1}^{k}\left(\xi_{i} \cdot y\right) \eta_{i}=y$ in $\sigma\left(E^{\prime}, E\right)$;

(5) $\Upsilon$ is total in $\sigma\left(E^{\prime}, E\right)$. 
Proof. Assume (1); we show that (2) follows. Consider first an $x$ which is a linear combination of $\xi$ 's. Let $X_{0}$ be the $\Upsilon$-orthogonal set given by Proposition 3. Then for $X>X_{0}, x=\sum_{1}^{\boldsymbol{k}}\left(x \cdot \eta_{i}\right) \xi_{i}$, and thus $x$ satisfies (2) trivially. Now consider a general element $x_{1}$ of $E$, and suppose it does not have property (2). Then there exist $\epsilon>0$, a cofinal family of $\Upsilon$-orthogonal sets $\left\{X^{\prime}\right\}$, and for each $X^{\prime}$ a $Y^{\prime}$ biorthogonal to it, such that

$$
\left\|x_{1}-\sum_{1}^{k}\left(x_{1} \cdot \eta_{i}^{\prime}\right) \xi_{i}^{\prime} \cdot\right\| \geqq \epsilon
$$

for every pair $X^{\prime}, Y^{\prime}$. For each $X^{\prime}$, let $s_{X^{\prime}}(x)=\sum_{1}^{k}\left(x \cdot \eta_{i}^{\prime}\right) \xi_{i}^{\prime}$ (singlevalued, since we have a unique $Y^{\prime}$ for each $\left.X^{\prime}\right)$. $s_{X^{\prime}}$ is a continuous linear mapping of $E$ into itself. It follows from the quasi-regularity that in the space $\mathcal{L}(E, E)$ of linear continuous mappings of $E$ into itself, the set $\left\{s_{X^{\prime}}\right\}, X^{\prime}$ running through the cofinal family above, is bounded in the topology of pointwise convergence. Since $E$ is complete, this gives us that the set $\left\{s_{X^{\prime}}\right\}$ is equicontinuous $[2$, Theorème I]. Now from the first part of the proof, $\lim \prec\left\|x-s_{X^{\prime}}(x)\right\|=0$ for every $x$ which is a linear combination of $\xi$ 's. It follows from the equicontinuity that the same is true for all $x \in E[3$, p. 29, Proposition 3], which contradicts (i) above.

Now (2) of course implies (3), and since (3) and (4) are each equivalent to

(6) For each $x \in E, y \in E^{\prime}, \lim \prec \sum_{1}^{k}\left(x \cdot \eta_{i}\right)\left(\xi_{i} \cdot y\right)=x \cdot y$,

they are equivalent to each other.

Now assume (4) and suppose $\mathcal{X}$ is not total. Then there exist $x \in E$ and $y \in E^{\prime}$ such that $y(\xi)=0$ for all $\xi$ and $y(x) \neq 0$. It follows that $\lim \prec \sum_{1}^{k}\left(\xi_{i} \cdot y\right) \eta_{i}=\theta$ (the neutral element) in $\sigma\left(E^{\prime}, E\right)$ while $y \neq \theta$. This contradicts (4).

The same line of argument gives us that (3) implies (5), which completes the proof.

Proposition 5. Suppose that to each $\xi \in X$ there is assigned a real number $\mu(\xi)$ such that

(a) If $\xi=\sum_{1}^{k} a_{i} \xi_{i}$, then $\mu(\xi)=\sum_{1}^{k} a_{i} \mu\left(\xi_{i}\right)$;

(b) The set $\left\{\sum_{1}^{\boldsymbol{k}} \mu\left(\xi_{i}\right) \eta_{i} \mid X, Y\right.$ biorthogonal $\}$ is bounded in $E^{\prime}$.

Then there exists $a y \in E^{\prime}$ such that $\mu(\xi)=y(\xi)$ for all $\xi$.

From (b) the set $\left\{\sum_{1}^{k} \mu\left(\xi_{i}\right) \eta_{i} \mid X, Y\right.$ biorthogonal $\}$ is contained in a subset $K$ of $E^{\prime}$ which is compact in $\sigma\left(E^{\prime}, E\right)$. For each $\xi$, let $K(\xi)$ denote the closure in $\sigma\left(E^{\prime}, E\right)$ of the set $\left\{\sum_{1}^{k} \mu\left(\xi_{i}\right) \eta_{i} \mid X>\xi, Y\right.$ bi- 
orthogonal to $X\}$. Each $K(\xi) \subset K$, and the family $\{K(\xi) \mid \xi \in X\}$ has the finite-intersection property. Hence, since $K$ is compact, $\bigcap_{\xi \in \mathcal{X}}$ $K(\xi)$ is nonempty. Choose $y$ from this intersection. We show $y$ has the required property. Consider a fixed $\xi . y$ is in $K(\xi)$, hence given $\epsilon>0$, we have $\left|\xi \cdot y-\xi \cdot\left(\sum_{1}^{k} \mu\left(\xi_{i}\right) \eta_{i}\right)\right|<\epsilon$ for some $X>\xi$ and $Y$ biorthogonal to $X$. Now $\xi=\sum_{1}^{k} a_{i} \xi_{i}$, therefore $\xi \cdot\left(\sum_{1}^{k} \mu\left(\xi_{i}\right) \eta_{i}\right)=$ $\sum_{1}^{k} a_{i} \mu\left(\xi_{i}\right)=\mu(\xi)$, from (a). Thus $|\xi \cdot y-\mu(\xi)|<\epsilon$ and since $\epsilon$ was arbitrary, $\xi \cdot y=\mu(\xi)$. This completes the proof.

If $X$ is total, the $y$ obtained in the above proposition is unique. This gives us the

CoRollary. If $X$ is total there is a 1-1 correspondence between the elements of $E^{\prime}$ and the functions $\mu(\xi)$ satisfying conditions (a) and (b) in Proposition 5.

Thus, if $X$ is total, then under quasi-regularity the situation is relatively simple and is essentially described in the theorem and the corollary to Proposition 5. The obvious example from integration is the following. Let $\Upsilon=\{\eta\}$ be a set, $S$ a $\sigma$-ring of subsets of $\Upsilon$ which covers $\Upsilon, E$ the space of bounded real functions on $\Upsilon$ measurable $(S)$, and $X=\{\xi\}$ the collection of characteristic functions of members of $S$. We put the supremum norm on $E$. Then $E$ is complete and $X$ is total. We identify each $\eta \in \Upsilon$ with the linear functional on $E$ defined by it. Then the $\boldsymbol{Y}$-orthogonal sets of $\mathcal{X}$ are those corresponding to the finite collections of mutually disjoint members of $S$, and it is trivial that the sytem $(X, Y)$ is biorthogonal and quasi-regular.

In the theorem, (1) or (2) are essentially equivalent in the present example to the measurability $(S)$ of $x$. The corollary to Proposition 5 says that $E^{\prime}$ is the space of all finitely additive, bounded set functions defined on the members of $S$. And finally (3) and (4) in the theorem, in the more familiar form (6), are simply the statement that $y(x)$ is given by the integral $\int x d y$.

3. When $X$ is not total, the situation is of course more involved. For example, in place of the theorem, we have the following (see [1, Propositions 5 and 7$]$ ): Let us denote by $A$ the closed linear subspace of $E$ generated by $X$, and by $A^{0}$ the subspace of $E^{\prime}$ orthogonal to $A$. Then (2) of the theorem becomes

Proposition 6. Let the system $(X, \Upsilon)$ be quasi-regular. Then for each $x \in A$,

$$
\lim \prec\left\|x-\sum_{1}^{k}\left(x \cdot \eta_{i}\right) \xi_{i}\right\|=0 .
$$

And (4) becomes 
Proposition 7. Let the system $(X, \Upsilon)$ be quasi-regular. Let us denote by $\phi$ the natural homomorphism of $E^{\prime}$ onto $E^{\prime} / A^{0}$. Then for each $y \in E^{\prime}, \lim <\sum_{1}^{k}\left(\xi_{i} \cdot y\right) \phi\left(\eta_{i}\right)=\phi(y)$ in $\sigma\left(E^{\prime} / A^{0}, A\right)$.

Most of the definitions and propositions of Dieudonné's paper carry over to the present paper if quasi-regularity is added to the conditions. We remark at this point that quasi-regularity as defined here is stronger than that defined by Dieudonné; it is in fact property (1) of his Proposition 16. However because of the many-valued relation between the $\xi$ 's and the $\eta$ 's, it does not imply convergence of the directed system $\left\{\sum_{1}^{k}\left(\xi_{i} \cdot y\right)\left(x \cdot \eta_{i}\right)\right\}$ for each $x, y$, whereas in his case it does.

Indeed, because of this same reason of many-valuedness, it turns out that, for the study of spaces of bounded functions, the later definitions and propositions in [1] are largely vacuous, and apparently a different approach is required.

\section{BIBLIOGRAPHY}

1. J. Dieudonné, On biorthogonal systems, Michigan Mathematical Journal vol. 2 (1953-1954) pp. 7-20.

2. N. Bourbaki, Sur certains espaces vectoriels topologiques, Annales de l'Institut Fourier vol. 2 (1950) pp. 5-16.

3. - Eléments de mathématique, Book III: Topologie genérale, Chap. X, Actualitiés Scientifiques et Industrielles, no. 1084, Paris, Hermann, 1949.

4. S. Banach, Theorie des operations linéaires, Warsaw, 1932.

WAYNE UNIVERSITY 